\title{
Sachsens Wirtschaft im Wandel 1990 bis 2015
}

\author{
Konstantin Hermann
}

Konstantin Hermann/André Thieme: Sächsische Geschichte im Überblick, Leipzig 2013, S. 214.

2 Siehe folgende Erscheinungen zur Wirtschaftsgeschichte Sachsens nach 1990: Günter Krause: Wirtschaftstheorie in der DDR. Marburg 1998; Ulrich Heß: Unternehmer in Sachsen. Aufstieg - Krise - Untergang - Neubeginn. Leipzig 1998; ders.: Unternehmen im regionalen und lokalen Raum 1750-2000. Leipzig 2004; Rainer Karlsch/Michael Schäfer: Wirtschaftsgeschichte Sachsens im Industriezeitalter. Leipzig 2006; Michael Schäfer: Schocktherapie und Leuchtturmbau. Wirtschaft in Sachsen nach 1990. In: Konstantin Hermann (Hrsg.): Sachsen seit der Friedlichen Revolution. Tradition - Wandel - Perspektiven. Beucha 2010, S. 173-183.

3 Sieh dazu die beiden Statistiken: http://www.khd-research. net/Docs/BAfA_Arbeitslose 1991-2007.pdf und http:// de.statista.com/statistik/daten/ studie/2522/umfrage/entwicklung-der-arbeitslosenquote-insachsen-seit-1999/ (letzter Abruf am 21.8.2015).
Eine Geschichte der sächsischen Wirtschaft nach 1990 kann nicht erst nach der Wende beginnen, aber ebenso nicht erst 1949 oder 1945. Eine Plattitüde, gewiss, aber eben doch von nicht geringer Relevanz für die Entwicklung der Nachwende-Ökonomie. Das Grundgesetz der Bundesrepublik Deutschland sieht die Herstellung gleichwertiger Lebensverhältnisse vor. Eine der Grundlagen dafür ist eine stabile wirtschaftliche Situation in den Regionen, die dies ermöglicht. Die Ausgangsbedingungen dafür waren jedoch weder in den neuen Ländern insgesamt noch in Sachsen selbst gleich. Das Territorium des 1990 wiedergegründeten Freistaats wies auch in der DDR große Industriezentren auf. Einige Ursachen dafür liegen Jahrhunderte zurück und sind schnell zu skizzieren: der Bergbau, der zu zahlreichen Entwicklungsschüben in diversen Gewerken und $\mathrm{zu}$ großen Einnahmen des Landesherren führte, wenn auch nicht in dem Maße wie früher oft kolportiert, die durch Kurfürst August von Sachsen (1526-1586) erlassenen Vorgaben zu Landwirtschaft und zur Wirtschaft sowie die Rolle Leipzigs als Messestadt und Dresdens als Residenz mit allen damit verbundenen Wirkungen. Dresdens Bedeutung als Zentrum der Nahrungs- und Genussmittelindustrie rührt ebenso daher wie als das der Feinmechanik und Pharmazie. Die Langzeitwirkungen sächsischer Geschichte beziehen sich also auch auf die Wirtschaft. In den flussreichen Gegenden des Erzgebirges und des Vogtlandes hatten sich die Textil- und Maschinenbauindustrie angesiedelt. Vor 1945 bestanden in Sachsen mittelständische Betriebe, die bis heute als Unternehmensform Deutschlands ökonomisches Rückgrat bilden, aber auch Konzerne, wenn sie auch nicht mit der AEG oder Krupp und anderen zu vergleichen waren. Träger dieser Wirtschaft waren die Angehörigen des Unternehmerbürgertums, aber besonders im 19. Jahrhundert die sozialen Aufsteiger, die Gewinner der Industrialisierung, denen sich die Chance - ähnlich wie heute durch die Digitalisierung - auf ein schnelles finanzielles und gesellschaftliches Emporkommen eröffnete. Meist stammten sie aus Handwerkerfamilien, waren Mechaniker, technikaffin, und gründeten Betriebe. Die Unternehmen wuchsen und wurden vererbt. Die Villen der Eigentümer, oft auf oder am Werksgelände, zeugen noch heute von dem geschaffenen Wohlstand. Sachsen als Erfinderland - ein Mythos, der nicht der Grundlage entbehrt - ist heute wieder zu einem Werbeträger geworden. Das Klischee vom „gemiedlichen Sachsen“, des wegen seines Dialekts vertrottelt Dargestellten, passte und passt nicht zu der Wirtschaftskraft dieses Landes. In Westdeutschland ist kaum bekannt, welchen ökonomischen Stellenwert Sachsen vor 1945 innerhalb Deutschlands einnahm; nicht selten sieht die westdeutsche Öffentlichkeit das Bestreben der Landesregierung, Sachsen auf der Seite der Geberländer des Länderfinanzausgleichs zu positionieren, als bloße Nachahmung von Bayern und BadenWürttemberg an. Dass es sich „lediglich“ um die Wiedergewinnung einer früheren wirtschaftlichen Stärke handelt, wird schnell übersehen. Aber das gilt auch für die Bezeichnung „Freistaat“, die allzu gern als Nachmacherei gilt, obwohl Sachsen früher Freistaat war als manch anderes Land, das sich mit dem Namen heute schmückt. Aber nicht nur personell oder strukturell, sondern auch das Verständnis von Wirtschaft selbst unterlag einer starken Transformation nach 1990. Anders als in demokratischen Staaten, wo die Politik per se das Primat hat, war der Stellenwert von Politik und Ökonomie in den sozialistischen Ländern unterschiedlich. Dies lag - neben sachlichen Zwängen - an den uneinheitlichen Auffassungen der „sozialistischen Klassiker“, die zugunsten des einen, aber auch des anderen interpretiert werden konnten.

\section{Ausgangsbedingungen}

Sozialistische Ökonomie beinhaltete ein Gutteil Sozial- und damit staatlicher Selbstsicherungspolitik, wohlverstanden Selbstsicherung. Nach 
dem 17. Juni 1953 musste die Wirtschaft dafür sorgen, dass die Interessen der Bevölkerung befriedigt werden konnten, koste es, was es wolle. Die soziale Destabilisierung hätte unweigerlich $\mathrm{zu}$ einer politischen geführt. So kam den Betrieben die Rolle $\mathrm{zu}$, allumfassend $\mathrm{zu}$ sorgen: die benötigten Waren nach den Vorgaben des Fünfjahresplans zu produzieren, soziale Versorgungs- und Sicherungssysteme vorzuhalten und letzten Endes die viel apostrophierte „Zweite Familie“, das Kollektiv, darzustellen. Der Verlust dieser sozialen und alltagskulturellen Eigenschaften der Betriebsstätte gehörte zu den wichtigsten Erfahrungen Ostdeutscher nach 1990, auch wenn es genügend gab, die versuchten, sich dieser „Fürsorge“ zu entziehen. DDR-spezifisch im Vergleich zur Bundesrepublik war der Anteil der erwerbstätigen Frauen. In Ostdeutschland arbeiteten 1989 über 90 Prozent der Frauen. Mitte der 1950er Jahre war die Frauenbeschäftigungsquote zwischen Westund Ostdeutschland noch fast gleich (ca. 50 Prozent), 1989 betrug sie in der Bundesrepublik nur unwesentlich mehr, 55 Prozent. Auch hier gab es nach 1990 Anpassungen. Heute arbeiten im Durchschnitt 70 Prozent der Frauen in ganz Deutschland. ${ }^{1}$

Sachsen wies nach 1990 für den Wirtschaftsumbau einerseits die besten, andererseits auch die schlechtesten Bedingungen auf. Der Maschinenpark war meist, bis auf wenige Betriebe wie Niles in Karl-Marx-Stadt, veraltet. Die Textilindustrie, in Westdeutschland aufgrund der billigen asiatischen Konkurrenz bereits viele Jahre vorher zusammengebrochen, gehörte zu den wichtigsten Wirtschaftszweigen in den drei Bezirken, vor allem im Bezirk Karl-Marx-Stadt. Aber auch sie geriet unter die Räder der globalisierten Wirtschaft. Heute ist nur noch ein Zwanzigstel in der sächsischen Textilindustrie

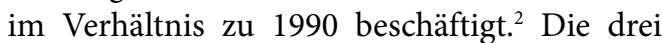
sächsischen Bezirke verfügten jedoch über technisch gut ausgebildete Menschen und eine trotz der Verstaatlichungen noch rudimentär erhalten gebliebene kleinteilige Wirtschaftsstruktur.

Seit dem 9. November 1989 konnten sich die DDR-Bürger von der Leistungsfähigkeit der westdeutschen Wirtschaft überzeugen; wer den Schilderungen der Westreisenden nicht geglaubt hatte, sah es nun selbst. Immer stärker strömten Westprodukte in die DDR. Zu dem Akzeptanzproblem der Ostprodukte, wie sie bald hießen, trat am 1. Juli mit der Einführung der DM ein knallhartes ökonomisches: Die starke Verteuerung ostdeutscher Produkte, ganz gleich, ob es die Waren täglichen Bedarfs waren oder wichtige Exportgüter, die nunmehr

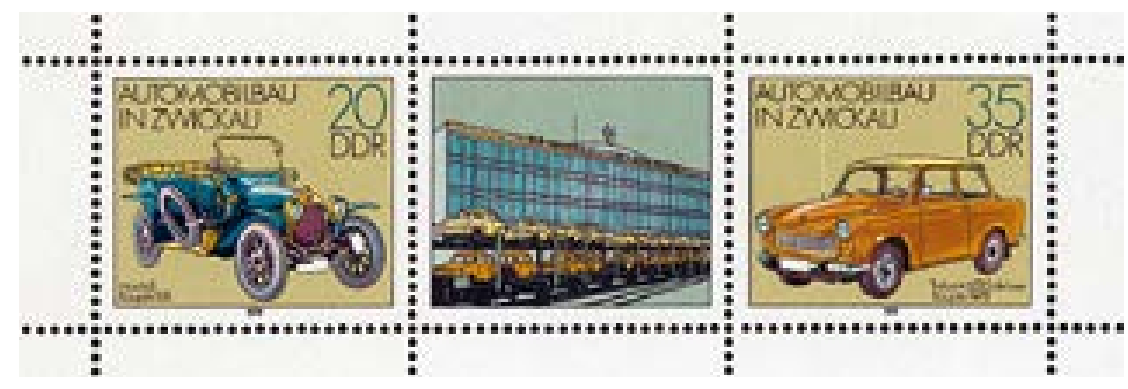

in DM zu bezahlen waren, was kaum eines der wichtigsten Handelspartner in Osteuropa konnte. Der Handel mit den Ostblockländern brach demzufolge ein. Die Belegschaften waren $\mathrm{zu}$ groß, die Produkte unter Marktbedingungen zu teuer, die Treuhandanstalt versuchte, die Betriebe zu privatisieren. Massenentlassungen und Betriebsschließungen waren die Folge. Arbeitslosigkeit zog in fast jeden ostdeutschen Haushalt ein. Ihren Höhepunkt erreichte sie in Sachsen - nach einem sprunghaften Anstieg bereits 1992 - im Jahr 1998 mit 18,8 Prozent und erreichte 2005 mit über 18 Prozent einen weiteren hohen Wert, während sie jetzt bei 8 Prozent liegt. Allerdings lag sie auch zwischen 1998 und 2005 permanent hoch. ${ }^{3}$ Nicht berücksichtigt sind in der Statistik die zahlreichen Maßnahmen wie Umschulungen usw. Die Gehälter waren weit von dem entfernt, was im Westen gezahlt wurde. Arbeitslosigkeit, Kurzarbeit, befristete Arbeitsverhältnisse und anderes sorgen dafür, dass diese Generation in wenigen Jahren vor massiven Problemen bei der Altersversorgung stehen wird.

Die Politik der Bundesregierung, die anfangs auf die Regulierungskräfte des Marktes setzte, ging von ihren Hoffnungen auf eine schnelle Angleichung der Lebensverhältnisse bald ab; man griff in den Markt ein. Sonderwirtschaftszonen, wie von manchen gefordert, richtete die Regierung jedoch nicht ein. Die Menschen verließen ihre Heimat und gingen in den Westen. Vor allem die Baubranche boomte in den Jahren nach der Wende und wurde der wichtigste Träger der Wirtschaft der ostdeutschen Länder. Als sie nach dem Ende der Bau- und Sanierungskonjunktur auf ein wirtschaftlich gerechtfertigtes Maß zurückging, fehlte den neuen Ländern ein wichtiges ökonomisches Standbein. Es war die Zeit der Glücksritter, wie Jürgen Schneider, die in ostdeutsche Immobilien investierten und einen Wechsel auf die Zukunft machten, der später nicht eingelöst werden konnte. Imperien, wie das von Schneider 1994, brachen zusammen; westdeutsche Anleger in Immobilien, oft getäuscht über die Vermietungs- und Vermarktungsaussichten, verloren wohl Millionen DM. Den Ostdeutschen wiede-
Automobilbau in Zwickau, Briefmarkenblock der Deutschen Post der DDR, 1979 
rum fehlte das nötige Kleingeld, um in solchen Klassen mitspielen zu können. Aber wie vieles hat auch dies zwei Seiten: Schneider investierte zielgerichtet in städtebaulich wertvolle Objekte, die zu wichtigen Ankerbauten der Sanierung wurden.

\section{Leuchttürme der Wirtschaft}

Der Umbau der sächsischen Wirtschaft nach 1990 war vor allem durch die „Leuchtturmpolitik“ bestimmt, die industrielle Wachstumskerne vorsah, um die herum sich weitere Unternehmen ansiedeln würden. Sie unterschied sich damit von den Ansätzen anderer neuer Länder, die nach dem „Gießkannenprinzip“ und unter falsch verstandener Gleichheit der Lebensbedingungen die Wirtschaftsleistung im Land verteilen wollte. Bestimmt wurde diese Politik durch den Ministerpräsidenten Kurt Biedenkopf und den Wirtschaftsminister Kajo Schommer (19402007). Anders als in den Ostländern bestand auch eine personelle Kontinuität: Schommer amtierte als Wirtschaftsminister von 1990 bis 2002. Die nachfolgenden Minister waren in der Regel nur eine Legislaturperiode im Amt. In derselben Zeit, in den ersten 12 Jahren nach der Wende, wechselten in Thüringen dreimal die Minister, in Sachsen-Anhalt sogar siebenmal, in Brandenburg viermal und in Mecklenburg-Vorpommern fünfmal. Diese Beständigkeit in Sachsen war die Folge der stabilen politischen Entwicklung, die die CDU in absoluter Mehrheit im Landtag von 1990 bis 2004 garantierte. Die positiven Folgen für die sächsische Ökonomie waren im Vergleich zu den anderen neuen Ländern bald zu sehen: Sachsen wies die niedrigste Arbeitslosenquote in Ostdeutschland auf, wenn sie im Vergleich zum Westen immer noch hoch war. Dies erscheint umso beachtlicher, als Sachsen aufgrund seiner geographischen Lage kein Pendlerland war: Anders als Thüringen, Brandenburg oder Sachsen-Anhalt verfügte es nicht über eine längere Grenze zu einem westlichen Bundesland oder Stadtstaat wie Berlin. Doch mental sahen sich viele weiter abgehängt: Man verglich sich nicht mit den anderen neuen Ländern oder gar dem Ostblock, sondern eben dem Westen. Manche Wahrheiten, die die Politiker sagten, führten zur Ablehnung und Wut. So auch von der fehlenden Perspektive vieler Langzeitarbeitsloser in den 1990er Jahren, die kaum mehr in Lohn und Brot zu bekommen seien.

Die sächsische Wirtschaft ist mittelständisch geprägt und entspricht damit der Form, wie sie hier vor 1945 entsprach. ${ }^{4}$ Wenn im folgenden von den Leuchttürmen der sächsischen Wirtschaft ge-schrieben wird, muss dabei der Mittelstand "mitgedacht“ werden, der sich in diesen
Regionen ansiedelt. Ebenso die regionale Gliederung: Während im Nordwesten Sachsens, im Leipziger Raum, vor allem der Dienstleistungssektor dominiert, ist es im Raum Erzgebirge/ Chemnitz die stark exportabhängige Produktion wie Maschinenbau und Elektroindustrie, in einigen Regionen der Oberlausitz die chemische Industrie. Daraus folgen verschiedene Konsequenzen von Wirtschaftskrisen, die in der Regel zunächst die stark exportabhängigen Unternehmen betrifft. Die Ansiedlung global agierender Unternehmen stellt sich aufgrund der nicht nur europa-, sondern weltweiten Konkurrenz als schwierig dar. Billigeren Produktions- und anderen Rahmenbedingungen muss sich Sachsen dabei stellen. Die alten Beziehungen, wie sie vor 1945 bestanden, waren durch die politischen Verhältnisse bald aufgelöst. Es braucht daher Zeit, neue Bindungen $\mathrm{zu}$ entwickeln. Gerade kleinen Unternehmen fällt dies jedoch schwer.

Eine Episode stellt dabei eine Personalie dar, die alte Konnexionen für neue wirtschaftliche Beziehungen nutzen sollte. Zum 1. Februar 2004 ernannte Wirtschaftsminister Martin Gillo den Betriebswirt und Kaufmann Alexander von Sachsen-Gessaphe, ein Urenkel des letzten sächsischen Königs, zum Ansiedlungsbeauftragten der sächsischen Regierung. Seit 1997 lebte er mit seiner Familie in Sachsen. Alexander sollte Investoren ins Land holen, vor allem aus Amerika. Interessant ist die Berufung des sächsischen Prinzen nicht wegen seiner Tätigkeit, sondern weil sich bei ihm ein bereits gewandeltes Beziehungsbild der Sachsen zu den Wettinern widerspiegelt. Hatten die Wettiner 1918, 1933, 1945 und 1989 in der Reputation der Bevölkerung gut und anerkannt überlebt, trat die Ernennung Alexanders von Sachsen zum Ansiedlungsbeauftragten auf eine geänderte Grundstimmung. Nun fiel auch die ehemalige Herrscherfamilie dem sozialistischen und nicht selten auch nach 1989 geübten Verdikt zum Opfer, das den Adel als Ausbeuter ansah. Gerade die linken Kräfte malten Drohkulissen über den heimkehrenden Adel an die Wand. Die Adligen hatten in der DDR keine Rolle mehr gespielt und wurden, da kaum noch Familienangehörige in Ostdeutschland wohnten, zu einer völligen Unbekannten, $\mathrm{zu}$ „Quasi-Außerirdischen“, von denen man vermutete, dass sie existieren, aber $\mathrm{zu}$ denen man keinen Bezug mehr hatte. Die Wettiner fielen aufgrund ihrer als unmäßig angesehenen Forderungen und der Verkäufe von wiedererlangtem Kunstgut nach den vier Vergleichen (1996, 1999, 2006 und 2014) dem Verdammnis der Bevölkerung anheim und verfügen in der öffentlichen Wahrnehmung über kein „positives Image“ mehr. Alexanders Ratschläge an die Sachsen 
bezüglich des „Benimms“ brachten ebenso wenig Gewinn wie seine eigentliche Tätigkeit als Ansiedlungsbeauftragter, die er bis 2008 ausübte. Ganz unrecht hatte er dabei nicht: Durch den Weggang des Groß- und mittleren Bürgertums mit den tradierten Konventionen des 19. Jahrhunderts, durch die Bevorzugung der „Arbeiter und Bauern" trat tatsächlich ein Verlust überlieferter bürgerlicher Kultur ein, den Alexander bemängelte. Durch den Weggang der Wirtschaftselite und die Verstaatlichung auch kleiner Betriebe fehlten unternehmerischer Geist und Risikobereitschaft - eine Langzeitfolge der DDRPolitik.

\section{Schwerpunkte der sächsischen Wirtschaft}

Der Blick auf die Produktion verstellt den Blick auf den hohen Anteil derer, die im Dienstleistungsgewerbe tätig sind. Im stark heterogenen tertiären Sektor sind über eine Million Sachsen beschäftigt, im primären 21.000 (Land- und Forstwirtschaft) und im sekundären 442.000 (Industrieproduktion). ${ }^{5}$ Der Tertiarisierungsgrad Sachsens entspricht damit dem Bundesdurchschnitt. Auch der Tourismus oder die Kreativwirtschaft sind wichtige Standpfeiler der Ökonomie. Wenn auch letztere nur im geringen Umfang zum Bruttosozialprodukt beiträgt, muss die Außenwirkung und ihre Funktion als Magnet für andere berücksichtigt werden. ${ }^{6}$ Im Tourismus boomen vor allem die beiden Städte Dresden und Leipzig, wo 40 Prozent aller Übernachtungen in Sachsen gebucht werden; aber letzten Endes profitiert jede Region von den Gästen. 2014 konnten über sieben Millionen Gäste in Sachsen begrüßt werden. ${ }^{7}$ Im produzierenden Gewerbe bilden sich einige Schwerpunkte $a b$, die die regional bezogene Leuchtturmfunktion haben; im Gegensatz zu den eben genannten Bereichen Tourismus, Land- oder Kreativwirtschaft.

Die Fahrzeugfertigung war ein wichtiger Bereich der Ökonomie im Süden der DDR geblieben: Trabant, MZ und Barkas kannte jedes Kind. Alle Konstrukteursideen in der DDR, den „Barkas“ oder den „Trabant“ weiterzuentwickeln, scheiterten an den fehlenden wirtschaftlichen oder auch politischen Voraussetzungen. Somit war der nötige Sprung in die Marktwirtschaft noch größer. Die sächsische Automobil- und Motorradproduktion nach 1990 kann durch die Entwicklung zweier Betriebe gekennzeichnet werden: der VEB Sachsenring mit dem „Trabant“ und die Motorradwerke Zschopau MZ. Letztere gingen 2012 endgültig in die Insolvenz, nach mehreren Besitzerwechseln und Namensumbe-

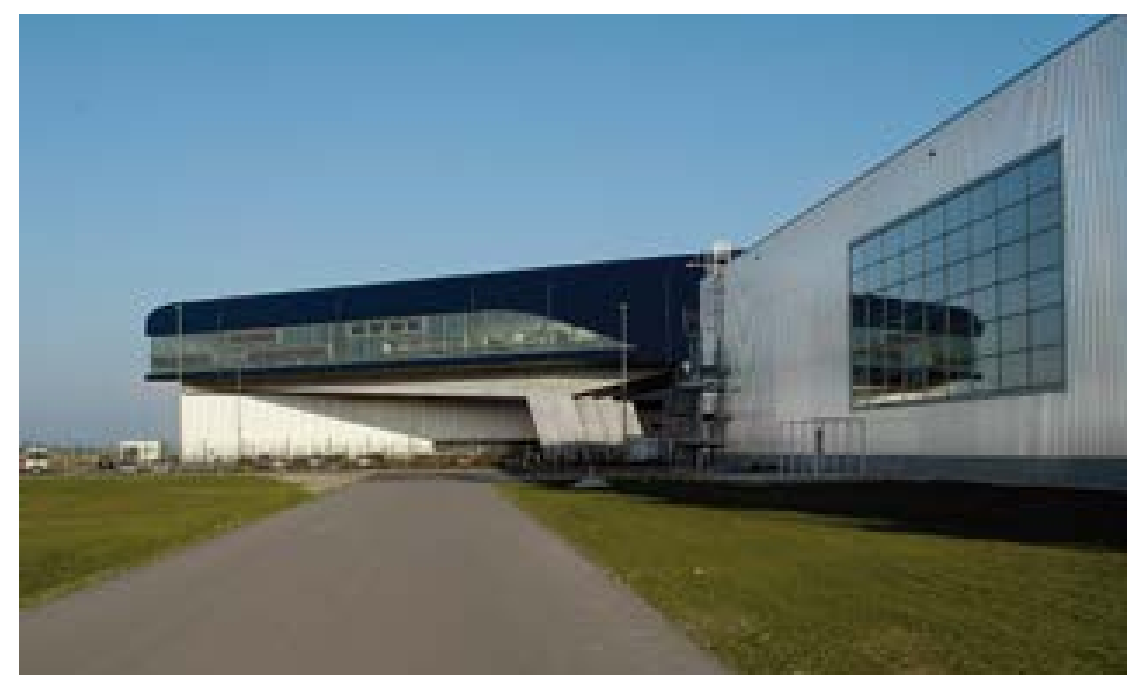

nennung. Es zeigt sich, dass die Kontinuität auch in der Wirtschaft ein hohes Gut ist. Dabei verfügte $M Z$ auch in Westdeutschland nicht unbedingt über einen schlechten Namen; zumindest war der Abstand zur Konkurrenz nicht so groß wie beim „Trabant“. Aber die VW-Werke Mosel, stabil geführt, gehören heute zu den wichtigsten sächsischen Unternehmensteilen, ähnlich wie BMW und Porsche, die sich später in Leipzig ansiedelten. In Dresden baute VW die „Gläserne Manufaktur“, eines der Paradebeispiele der „Eventkultur“ in der Industrie, die den Autokauf zum Erlebnis machen wollte. In der Manufaktur wird der Luxuswagen von VW produziert, der „Phaeton“. Über 700 Automobilbauzulieferer existieren heute in Sachsen. Die Barkas-Werke in Karl-Marx-Stadt haben die Nachwendezeit nicht überstanden.

Einer der weiteren Leuchttürme ist die IT-Branche in Dresden, hervorgehend aus dem Robotron-Komplex. Der VEB Robotron-Elektronik „Otto Schön“ Dresden produzierte seit den 1970er Jahren den Großrechner EC 1040, später andere Typen. Doch die Computerisierung im Westen war wesentlich weiter fortgeschritten. Ein letztes Aufbäumen, um an den Westen anzuschließen, war der 1-Megabit-Chip, den es im „NSW“ (Nichtsozialistischen Wirtschaftsgebiet) schon lange gab. Nach zweieinhalbjähriger Entwicklungszeit un-ter Beteiligung des Zentrums Mikroelektronik Dresden innerhalb des Kombinats Carl Zeiss Jena und anderer Einrichtungen konnte am 12. September 1988 der Chip an Erich Honecker (1912-1994) übergeben werden. Die DDR-Medien bejubelten diesen „Anschluss an die Weltspitze“. In Serie ging der Chip indes nicht mehr. Letzten Endes wirkten die Traditionen der Dresdner Feinmechanik und fotografischer Industrie in die Computerzeit fort, bis heute, denkt man an den VEB Schreibmaschinenwerk Erika, an den am 1. Januar 1968 gebildeten
BMW-Werk in Leipzig, Zentralgebäude Foto: BMW 
Pentacon und anderes. So konnten die sich für den Standort Dresden interessierenden Firmen auf ausgebildetes IT-Personal zurückgreifen. Infineon errichtete in Dresden 1995 ein großes Werk; weitere IT-Firmen siedelten sich an. „Silicon Saxony" hieß und heißt das Schlagwort einer Branche, die sich zumindest in Sachsen gegen die seitherigen Krisen gegenüber als recht robust behauptet hat. Zwar kostete die Insolvenz der Infineon-Tochter Qimonda im Jahr 2009 immerhin 4.000 Arbeitsplätze, aber der Standort Dresden erholte sich schnell. Auch andere Chiphersteller siedelten sich an; bisweilen unter massivem Einsatz von Steuergeldern. AMD, jetzt Globalfoundries, beschäftigt heute in Dresden über 3.700 Mitarbeiter. Insgesamt arbeiten heute über 50.000 Menschen in der sächsischen ITBranche.

Einen weiteren Schwerpunkt der sächsischen Wirtschaft stellen die Biotechnologie und die Pharmaindustrie in Leipzig und Dresden dar, mit starkem forschungsaffinen Charakter. Ein Höhepunkt dieser Entwicklung ist die Gründung des von der Fraunhofer-Gesellschaft etablierten „Nationalen Leistungszentrums Chemie und Biosystemtechnik“ 2014, die nochmals einen starken Schub auf diese Branche ausüben wird. „Bio-Saxony“ entstand vor allem nach der Jahrtausendwende, griff jedoch auch auf die Traditionen der sächsischen Pharma- und Chemieindustrie zurück. Die Solarbranche, die sich in und um Freiberg ansiedelte, steht seit 2012 vor einer unsicheren Zukunft. Zwar haben sich die
Verkäufe wieder stabilisiert, jedoch steht der Wirtschaftszweig durch die Konkurrenz aus China und durch die Reduzierung der finanziellen Förderung stark unter Druck. Immerhin stammt heute ein Viertel des sächsischen Stromverbrauchs aus erneuerbaren Energien. Der Preis dafür ist die „Bereicherung“ des Orts- und Landschaftsbilds mit Solar- und Windkraftanlagen. Durch den Rohstoffhunger entstand im Freiberger Raum, aber auch darüber hinaus im Erzgebirge ein Wiederaufleben bergbaulicher Vorhaben, auch wenn von einem neuen „Berggeschrei“ noch keine Rede sein kann. Der Abbau seltener Bodenschätze wird untersucht; zurückgegriffen wird dabei nicht selten auf Erkenntnisse aus der DDR. Welchen Umfang ein solches Projekt dereinst erreichen wird, hängt vor allem von der Entwicklung der Rohstoffpreise ab, die über die Rentabilität des Bergbaus entscheiden. Der landschaftsfressende Braunkohletagebau um Leipzig und in der Oberlausitz verlor an Bedeutung; wie so oft liegen Für und Wider eng beieinander. Noch ist die Zahl der Arbeitsplätze in den Tagebauen so hoch, dass sie wichtige Wirtschaftsstandorte sind; andererseits wiegen Heimatverlust und Umweltzerstörung schwer. Für die Bevölkerung in den Tagebauarealen bestand nach 1990 kurzzeitig Hoffnung, dass die Tagebauerweiterungen nicht stattfinden würden. Jedoch war dem Freistaat der Erhalt der Arbeitsplätze wichtiger. Nun scheint die Klimaschutzpolitik den Ausbau der Abbaggerung zu stoppen. Durch die Flutung der Tagebaue ent-
Globalfoundries in Dresden, Luftaufnahme Foto: fensterblick

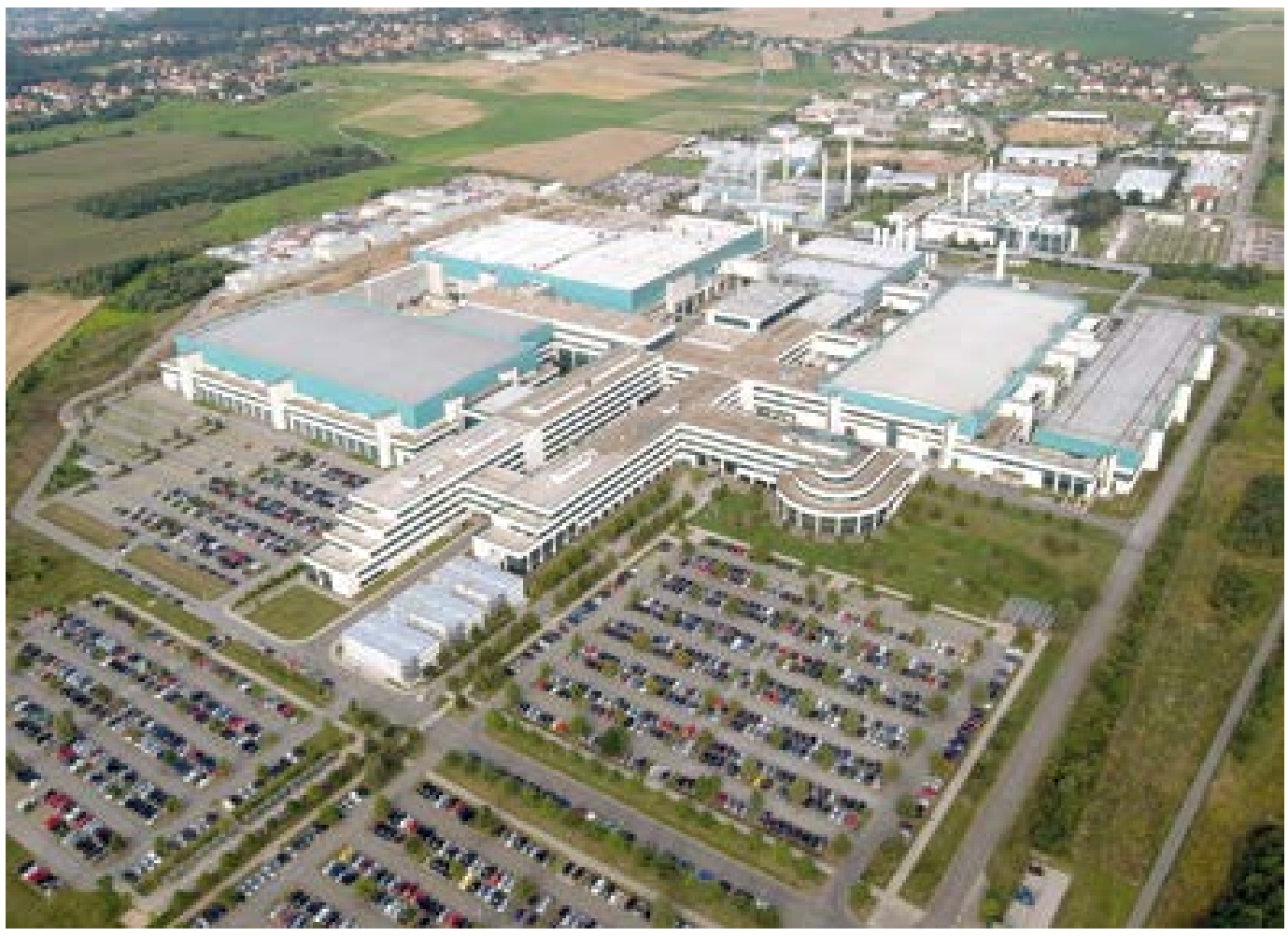


standen, nachdem sich Flora und Fauna wieder ansiedelten, attraktive Urlaubsregionen wie das Leipziger Neuseenland.

\section{Wirtschaft 4.0}

Ohne Zweifel waren die meisten der Entscheidungen der sächsischen Politik bezüglich der Wirtschaftspolitik richtig. Angesichts der vorhandenen Voraussetzungen konnten Fehler nicht vermieden werden - die, die gemacht wurden, vermögen jedoch nicht die Gesamtleistung signifikant $\mathrm{zu}$ schmälern, auch wenn sie schmerzhaft waren und Geld kosteten und kosten. Als 2007 die Landesbank Baden-Württemberg die Landesbank Sachsen aufgrund von Liquiditätsproblemen übernehmen musste, bürgte der Freistaat mit 2,75 Milliarden Euro. Es dauerte ein Dreivierteljahr, bis der sächsische Ministerpräsident Georg Milbradt als Folge der Landesbankkrise seinen Rücktritt erklären musste. Es wäre auch in Sachsen angesichts der bundespolitischen Rahmenbedingungen nicht möglich gewesen, den Umbruchprozess der Wirtschaft deutlicher abzufedern, was zum Beispiel Wirtschaftsminister Schommer mit seiner Rettung von Betrieben gegenüber der Treuhand versuchte. Manches konnte bewahrt werden, vieles nicht. So gelang es der sächsischen Regierung und der Stadt Leipzig mit weiteren Partnern, die Leipziger Messe in die Zukunft zu retten, die sich aufgrund der im Westen etablierten Messen starker Konkurrenz erwehren musste,

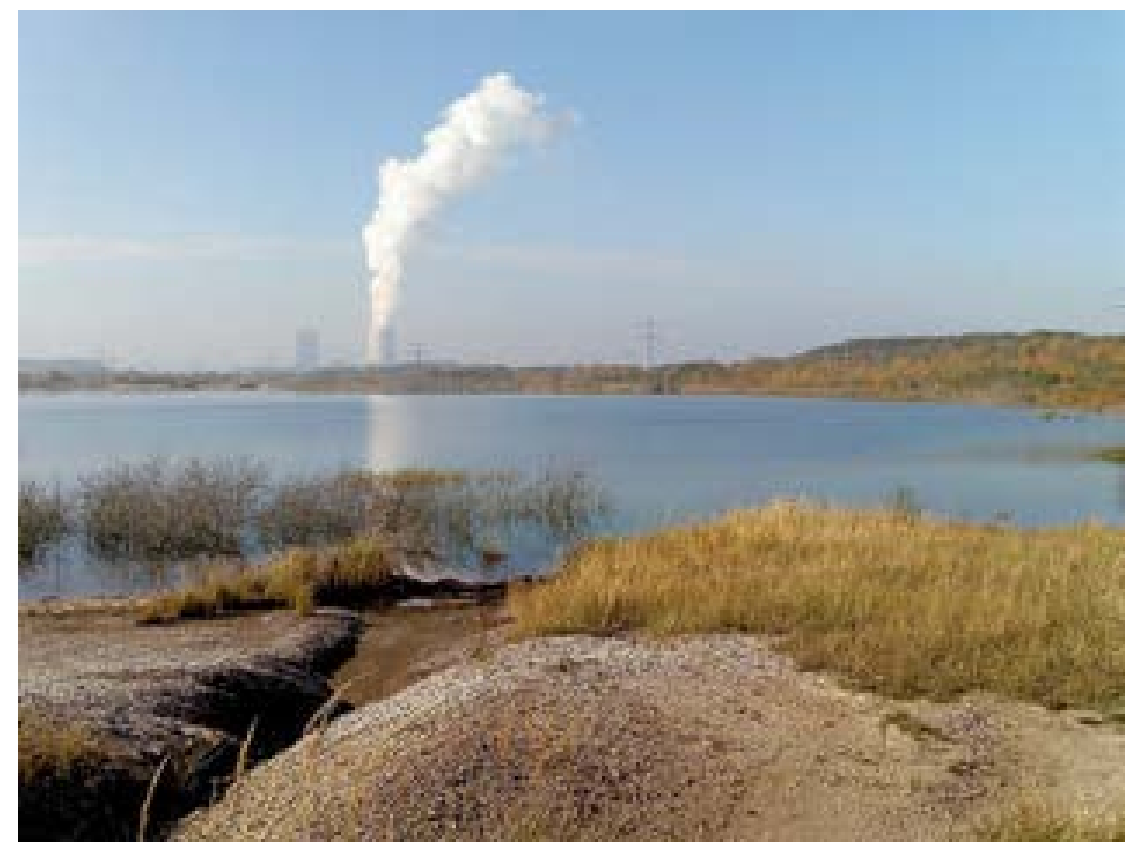

die nicht selten - gerade wie die Hannover-Messe - damals von exilierten Sachsen aufgebaut worden war. Der Messe-Markt war aufgeteilt; zahlreiche Fachmessen zogen das interessierte Publikum an. Die Leipziger Messe, in der DDR das wirtschaftliche Schaufenster, war nach 1990 in seiner Existenz bedroht; eine Messe, die vom Eierkocher bis zur Maschinenbauanlage alles präsentierte, musste neu erfunden werden. Um die Leipziger Messe herum wurden zahlreiche Fachmessen etabliert. Der Messestandort Leipzig ist heute gesichert.

Der Kahnsdorfer See, ein rekultivierter Braunkohlentagebau südlich von Leipzig, im Hintergrund das Kraftwerk Lippendorf Foto: wikimedia, Jwaller

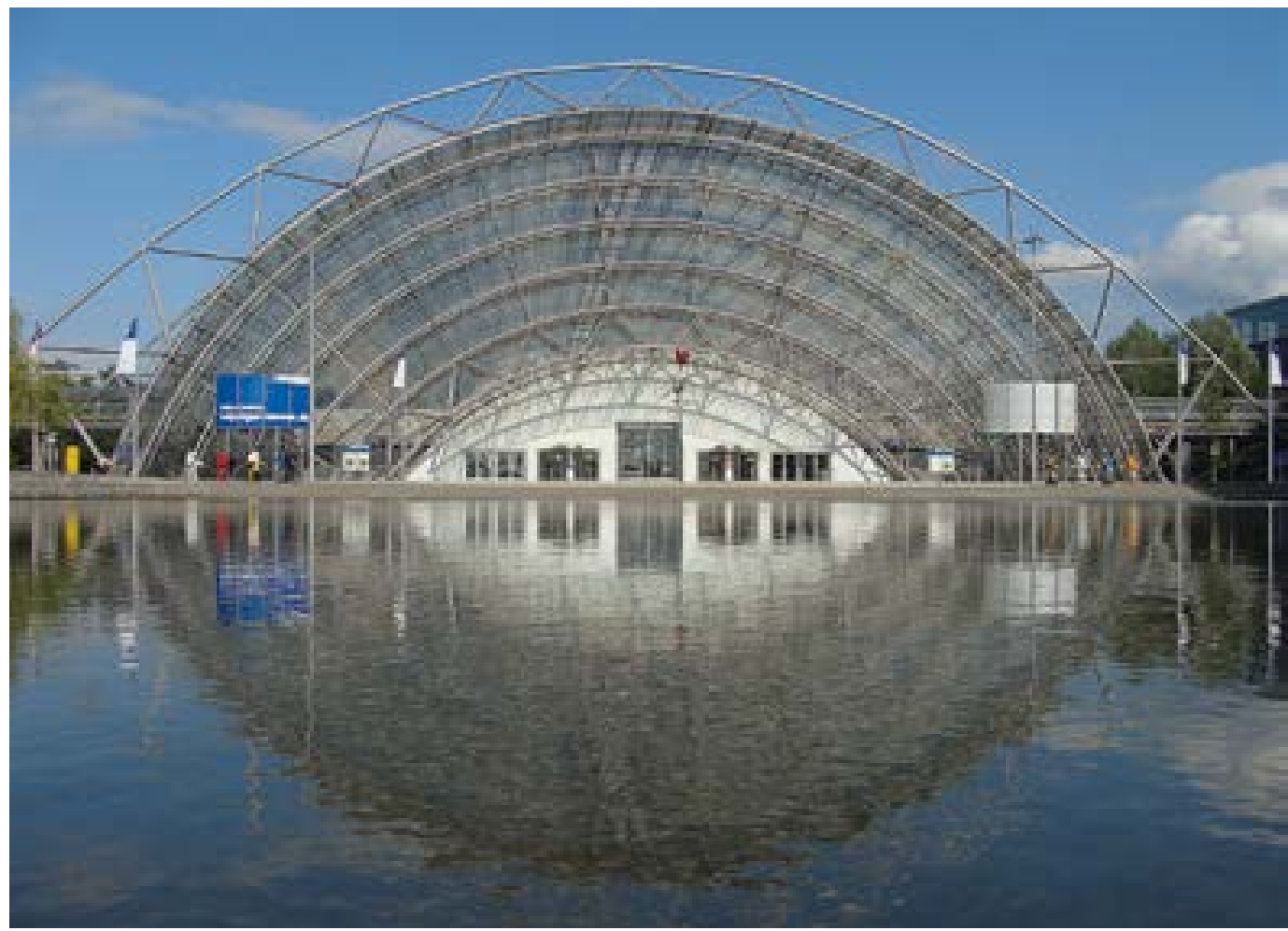


8 Siehe dazu: http://invest.dresden.de/de/Kompetenzfelder Branchen/Themenexzellenz/ Industrie_40_2314.html (letzter Abruf am 21.8.2015)

Gläserne Manufaktur in Dresden Foto: wikimedia, X-Weinzar

\section{Autor}

Dr. Konstantin Hermann

1. Vorsitzender des

Vereins für sächsische

Landesgeschichte und

Vorstandsmitglied des

Landesvereins Sächsischer

Heimatschutz

Sächsisches Staats-

ministerium für

Wissenschaft und Kunst

Wigardstraße 17

01097 Dresden
Die sächsischen Unternehmen standen und stehen seit 1990 vor einem dreifachen Umbruch: Einerseits mussten sie, oftmals ohne großes Eigenkapital, aufgebaut werden und standen im Wettbewerb mit den etablierten westdeutschen Firmen; zweitens setzte der Prozess der "Globalisierung“ ein, der zwar schon viel weiter zurückreicht, aber seit den 1990er Jahren das ökonomische Leben bestimmt, und drittens die Digitalisierung der Wirtschaft, die Industrie 4.0 - die Verzahnung des Internets und der Produktion, die „Künstliche Intelligenz". ${ }^{8}$ Gerade diese Entwicklung wird die Wirtschaft nochmals revolutionieren, nach der Einführung von Roboter- und Computertechnik. Nicht auszuschließen, dass sich auch durch den 3D-Druck eine Herausforderung ergibt: Wenn diese Technik im Alltag angekommen ist und jeder einen 3D-Drucker wie heute einen Papierdrucker im Haus hat, kann dies erhebliche Auswirkungen auf so manchen Hersteller haben, bis hin zum Lebensmittelhandel. Die Differenzierung nimmt durch die Digitalisierung zu: Der Internethandel, auch mit Lebensmitteln, steigt und eröffnet neue Kunden-Hersteller-Beziehungen. Die Wirtschaft benötigt, gerade im ländlichen Raum, den Ausbau schneller Internetverbindungen, was ansonsten schnell zu einem Standortnachteil avanciert.

Das Ende der EU-Regionalförderung für die neuen Länder ist abzusehen. Wenn die EFREPeriode 2020 endet, dürften die künftigen Empfängerländer weiter im Osten liegen. Hinzu treten noch die innerdeutschen Entwicklungen, die die Wirtschaft betreffen. DAX-Konzer- ne werden sich voraussichtlich auch in Zukunft nicht mit ihren Zentralen in Sachsen ansiedeln; die Kreativszene mit starken Startups findet in den Metropolen statt: Berlin, Hamburg, München, Köln. Es bleibt damit der Mittelstand auch als zukünftiger Träger der sächsischen Ökonomie, regional verankert, aber mit Handelsbeziehungen weit über die Region, bis hin in die ganze Welt, hinaus. Dabei wird auch die Rolle der Wissenschaft wichtiger. Von den Beschäftigten im Maschinenbau ist nur noch ein Viertel der Beschäftigtenzahl gegenüber 1990 übrig. Um so wichtiger werden Impulse und Zusammenarbeit der Wissenschaft, wie sie durch die Technischen Universitäten Dresden und Chemnitz gegeben werden. Besonders das Miteinander von Wissenschaft und Wirtschaft ist von hohem Stellenwert. Die Produktivität reicht indes bisher nicht aus, die westdeutschen Länder einzuholen, auch 25 Jahre nach der deutschen Einheit nicht. Mit knapp 70 Prozent Produktivitätsrate im Vergleich zum Westen hinkt der Freistaat, wie die anderen neuen Länder auch, 30 Jahre hinterher. Der Fachkräftemangel, den es in einigen Branchen gibt, verschärft dieses Problem noch genauso. Bezahlungen unterhalb des Tarifs dürften damit immer weniger möglich sein. Sachsen investiert in die Zukunftstechnologien. Der Freistaat agiert dabei unter starkem Konkurrenzdruck, unter den verändernden Bedingungen der Digitalisierung. Die Stärke der Regionen zu nutzen, ohne in Provinzialismus zu verfallen, dürfte auch in der Zukunft wie bisher der erfolgreichste Weg sein.

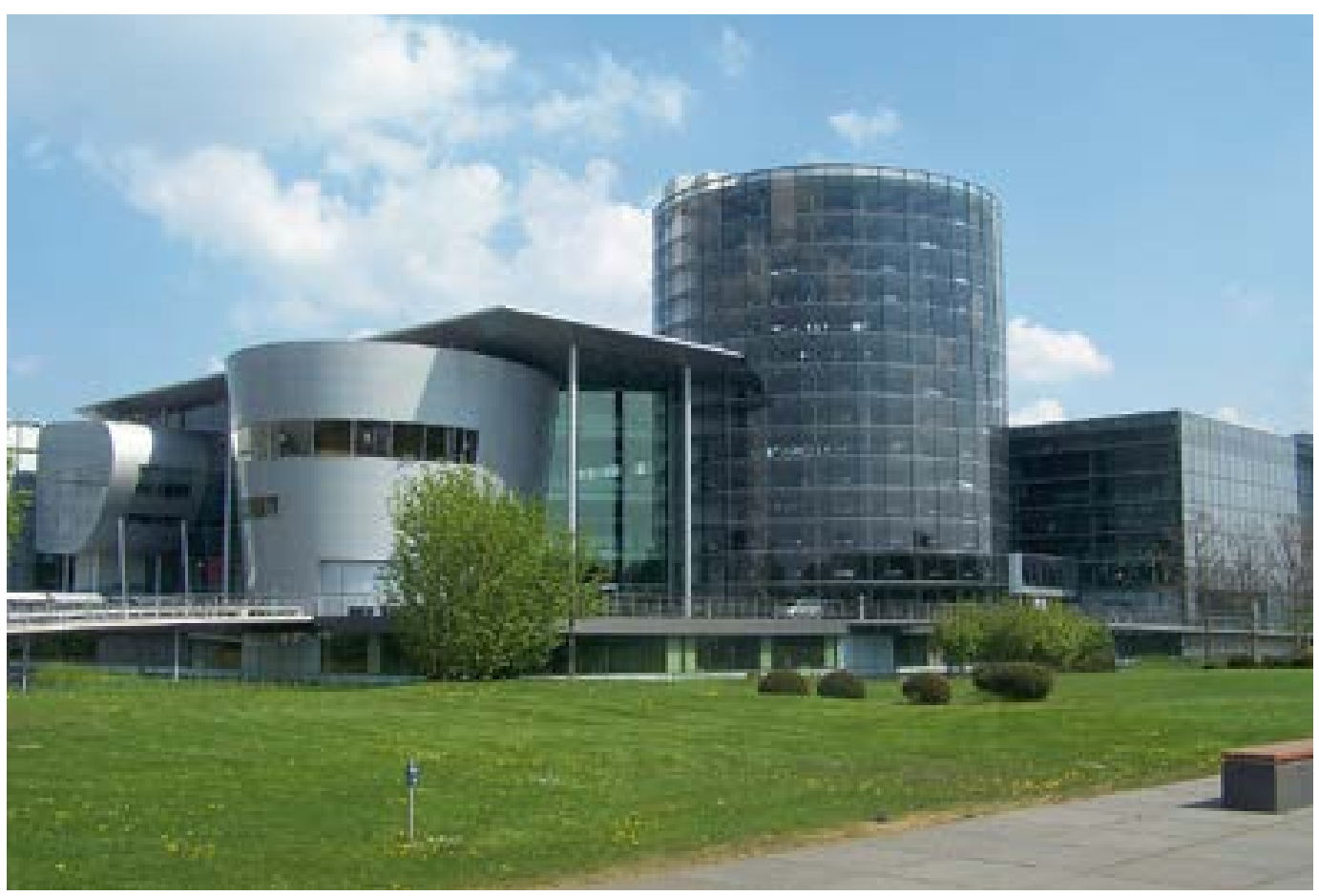

